\title{
Problemas sexuais na mulher com cancro da mama e cancro ginecológico: revisão narrativa
}

Maria Beatriz Morgado

\section{RESUMO}

Objetivos: Estima-se que mais de metade das mulheres com cancro da mama ou cancro ginecológico apresenta problemas sexuais. Contudo, apenas uma minoria recebe aconselhamento médico nesse âmbito. A presente revisão narrativa tem como objetivo descrever os principais problemas sexuais das mulheres com esta tipologia de neoplasias, bem como identificar estratégias de abordagem e tratamento dos mesmos.

Métodos: Foi efetuada uma pesquisa da literatura na base de dados PubMed, utilizando os termos MeSH: Sexuality, Neoplasms, Female e Women. Foram utilizados livros e artigos datados entre 1998 e 2020.

Resultados: O cancro da mama afeta a vida sexual desde o diagnóstico, associando-se a intenso sofrimento psicológico. A este acresce o impacto do tratamento. A cirurgia altera a autoimagem, sensibilidade mamária e mobilidade. A radioterapia condiciona alterações tecidulares. A quimioterapia apresenta efeitos psicológicos e físicos com impacto direto e indireto na sexualidade. A hormonoterapia condiciona atrofia urogenital e sintomas vasomotores. Por outro lado, também a maioria das mulheres com cancro ginecológico apresenta vários níveis de disfunção sexual secundários à terapêutica. Tratamentos mais recentes como a braquiterapia, técnicas de nerve-sparing modificadas e cirurgia reconstrutiva têm contribuído para uma diminuição da morbilidade associada ao tratamento. Verifica-se uma consciencialização crescente da importância da saúde sexual dos doentes oncológicos. Neste contexto, cabe ao médico abordar esta temática, informando as doentes acerca do possível impacto da doença e do respetivo tratamento na sexualidade. Existem questionários validados e modelos estruturados facilitadores de comunicação neste sentido. Cabe também aos clínicos o controlo dos sintomas de disfunção sexual associados à terapêutica oncológica, privilegiando sempre que possivel fármacos com impacto neutro ou positivo na sexualidade.

Conclusão: Qualquer médico deve estar sensibilizado para a disfunção sexual associada à patologia oncológica e ao seu tratamento, sendo capaz de a rastrear e adaptar o plano terapêutico em conformidade, colocando em prática o cancer care continuum.

Palavras-chave: Sexualidade; Oncologia; Neoplasia; Mulher.

\section{INTRODUÇÃO}



Organização Mundial da Saúde define saúde sexual como um estado de bem-estar físico, mental, emocional e social em relação à sexualidade, constituindo esta última um direito e um dos componentes major de uma vida saudável e satisfatória. ${ }^{1-3}$

O aumento da esperança média de vida associa-se a um aumento da incidência de neoplasias. ${ }^{4}$ Verifica-se também um aumento da sobrevida dos doentes oncológicos, condicionando um aumento da prevalência

1. Médica Interna de Medicina Geral e Familiar. USF Cova da Piedade, ACeS Almada-Seixal. Cova da Piedade, Portugal. desta classe de doenças - atualmente consideradas crónicas. ${ }^{4}$ Torna-se, assim, imperativo promover a melhoria da qualidade de vida desta população. ${ }^{5-8}$

Verifica-se uma elevada prevalência de problemas sexuais em doentes oncológicos, cujas estimativas variam entre os $40 \%$ e os $100 \% .{ }^{3,9-10}$ Contudo, pouco ênfase tem sido dado a esta realidade. ${ }^{3}$ Os doentes têm receio que a sexualidade não seja um assunto prioritário do ponto de vista clínico. ${ }^{3}$ Não abordam a sexualidade por constrangimento e por receio de deixarem o médico desconfortável, esperando que seja o profissional a abordar o tópico. ${ }^{11}$ Já os profissionais de saúde não abordam a sexualidade por escassez de tempo, falta de privacidade, conhecimento ou treino insuficientes, 
desconhecimento de estratégicas eficazes ou recearem causar constrangimento ao doente..$^{3,10-12}$ Adicionalmente, existe a ideia de que a sexualidade não é uma prioridade para um doente cuja patologia de base coloca em risco a sua sobrevivência. ${ }^{3,5}$ Num estudo que avaliou este aspeto, a maioria dos doentes oncológicos (74\%) considerou importante discutir os problemas sexuais com os profissionais de saúde que os acompanham, mas poucos haviam recebido qualquer informação por parte dos mesmos. ${ }^{13}$

Urge compreender-se a qualidade de vida como um fator primordial na avaliação do doente oncológico. ${ }^{14} \mathrm{~A}$ presente revisão narrativa tem como objetivo descrever os principais problemas sexuais das mulheres com cancro da mama e cancro ginecológico. Adicionalmente, identificar-se-ão estratégias para controlo dos sintomas mais frequentemente associados à disfunção sexual nesta população e serão descritas técnicas comunicacionais facilitadoras da abordagem à sexualidade.

\section{MÉTODOS}

Foi realizada uma revisão narrativa da literatura, utilizando a base de dados PubMed, com os termos MeSH: Sexuality, Neoplasms, Female e Women. Foram incluídos livros e artigos publicados em língua portuguesa e inglesa, disponíveis (texto integral), datados entre 01 de fevereiro de 1998 e 01 de maio de 2020, que visassem a população do sexo feminino, de idade adulta (igual ou superior a 18 anos), com diagnóstico estabelecido de cancro da mama ou cancro ginecológico (cancro do ovário, do endométrio, do útero, da vulva ou da vagina). Excluíram-se artigos e livros que abordassem patologia oncológica sem origem na mama ou nos órgãos ginecológicos femininos.

\section{RESULTADOS}

\section{A sexualidade do doente oncológico}

O impacto da doença oncológica na sexualidade começa no dia em que o doente recebe o diagnóstico. ${ }^{15} \mathrm{O}$ forte impacto emocional que o diagnóstico de uma doença oncológica representa conduz à inibição sexual ("novo eu") e a vida sexual do casal é frequentemente colocada em segundo plano ("novo nós"). ${ }^{15}$

Para manutenção da intimidade sexual satisfatória são necessários: controlo oncológico, capacidade físi- ca, disponibilidade emocional e psicológica. ${ }^{15}$ Os doentes que vêm abordados todos estes parâmetros pelos profissionais de saúde que os acompanham são uma população melhor tratada. ${ }^{15}$

De forma holística, cabe aos profissionais de saúde que acompanham doentes com patologia oncológica:

- Salvaguardar a permissão para abordar o tema da sexualidade e fornecer informação clara e limitada (de acordo com o que o doente pretende saber) sobre a forma como a doença oncológica e o seu tratamento podem afetar a sexualidade. ${ }^{3}$ Explicar os componentes do desejo/excitação/orgasmo, corrigindo mitos e ideias erradas sobre a fisiologia e a patologia. ${ }^{3}$ Uma vez informado, o doente estará melhor preparado para enfrentar as possíveis dificuldades futuras.

- Fornecer informação sobre a doença, incluindo o seu possível impacto a nível psicológico, e explorar estratégias para a resolução de problemas. ${ }^{16}$

- Informar o doente sobre as terapêuticas farmacológica e não farmacológica disponíveis para as disfunções sexuais e compreender a abertura da pessoa (ou casal) para as mesmas. ${ }^{3}$ Deverá priorizar-se a prestação de cuidados por equipas especializadas multidisciplinares, que poderão incluir a oncologia, ginecologia, urologia, cirurgia geral, cirurgia plástica e reconstrutiva, profissionais na área da saúde mental, medicina física e reabilitativa, centros especializados em técnicas de preservação da fertilidade, terapia de casal e/ou sexologia clínica. ${ }^{17}$

- Não «patologizar»e promover a readaptação do doente. ${ }^{3,18}$ Dever-se-á dar oportunidade ao doente de reinventar a sua sexualidade, de acordo com as suas possibilidades e expectativas atuais. ${ }^{19}$

\section{A mulher com cancro: impacto da doença oncológica na resposta sexual feminina}

A resposta sexual fisiológica é habitualmente descrita em quatro fases: o desejo, a excitação sexual, o orgasmo e a resolução. ${ }^{20-21}$ No homem, a resposta sexual costuma ser um processo linear que segue as fases supramencionadas; já na mulher isso nem sempre ocorre, podendo existir excitação sexual sem a fase precedente do desejo (quando o contacto erótico é iniciado pelo/a parceiro/a) e podendo existir satisfação sexual sem orgasmo mais frequentemente do que no homem.,21 
A disfunção sexual é uma condição multifatorial, para a qual contribuem componentes biológicos, psicológicos, sociais e culturais. ${ }^{3}$ Segundo uma meta-análise, a prevalência de disfunção sexual em doentes com cancro da mama poderá ascender aos $65,54 \%$ e cancro ginecológico aos $78,44 \% .{ }^{22}$ Os problemas sexuais neste contexto podem ser graves e persistir com o tempo. ${ }^{9} \mathrm{~A}$ Tabela 1 resume a fisiologia da resposta sexual feminina, bem como os fatores associados à doença oncológica e respetivo tratamento que podem contribuir para a disfunção sexual em mulheres com cancro da mama e cancro ginecológico. ${ }^{3}$

\section{Cancro da mama}

O cancro da mama tende a ser diagnosticado em idades mais jovens do que a maioria das neoplasias, sendo a idade mediana de 61 anos. ${ }^{9}$ Em Portugal é a neoplasia feminina com maior incidência $(30 \%$ das neoplasias em mulheres).$^{14} \mathrm{~A}$ sobrevivência a um ano é de $95 \%$ e a sobrevivência a cinco anos é de $83 \%$, havendo, assim, uma população considerável de sobreviventes a esta patologia..$^{14}$

\section{Cancro da mama: o impacto do diagnóstico}

A mama é um órgão privilegiado da mulher, com particular significado pessoal e social, pelo que qualquer ameaça à sua integridade está associada a um grande sofrimento. ${ }^{15} \mathrm{~A}$ receção do diagnóstico de cancro da mama acarreta uma disfunção significativa, surgindo muitas vezes estados de desesperança, medo e depressão. ${ }^{23} \mathrm{~A}$ antecipação das alterações da imagem corporal, o receio da perceção do novo status de saúde por terceiros e o medo de rejeição pelo/a parceiro/a contribuem negativamente para o funcionamento sexual da doente..$^{3,24-25}$

\section{Cancro da mama: o impacto do tratamento}

O tratamento do cancro da mama é variável consoante as características individuais de cada caso, podendo passar por cirurgia, radioterapia, hormonoterapia e/ou quimioterapia. No entanto, todos estes tratamentos podem afetar negativamente a função sexual alguns dos quais de várias formas. ${ }^{3}$

Em relação à cirurgia, as consequências que mais comummente afetam a sexualidade são: alteração da autoimagem, perda de sensibilidade mamilar, dor lo- cal, linfedema (menos frequente com a técnica do gânglio sentinela), diminuição da força, limitação da mobilidade do membro homolateral e postura assimétrica. ${ }^{3,18,26-28}$ A realização de cirurgia reconstrutiva pode ter um impacto positivo, com melhoria da disfunção sexual e da sintomatologia depressiva., ${ }^{3,27}$

As consequências da radioterapia dependem da dose total de radiação, área irradiada, duração do tratamento, idade e status prévio da doente. ${ }^{9}$ Os efeitos adversos mais comuns são as reações cutâneas, as alterações da sensibilidade, o edema e a fibrose tecidular. Estes podem associar-se a dor crónica e alteração da mobilidade dos membros. ${ }^{3,26,29}$ A insónia e a fadiga, frequentemente presentes após a radioterapia, poderão também afetar negativamente o desejo sexual.,26,29

Aproximadamente $80 \%$ dos cancros de mama expressam recetores de estrogénio e $65 \%$ expressam recetores de progesterona. ${ }^{3}$ A hormonoterapia é muitas vezes utilizada nestes casos. ${ }^{3}$ Quando se utilizam fármacos que bloqueiam a ação do estrogénio (como o tamoxifeno), o bloqueio hormonal dar-se-á quer ao nível das células tumorais, quer ao nível sistémico, desencadeando sintomas de privação hormonal. ${ }^{26} \mathrm{~A}$ nível central poder-se-á verificar maior irritabilidade, sintomas depressivos e sintomas vasomotores, bastante comuns e com impacto negativo na sexualidade. ${ }^{3}$ Estima-se que cerca de $47,5 \%$ das doentes tratadas com tamoxifeno apresentem sintomas vasomotores, mais prevalentes nos primeiros seis meses de tratamento. ${ }^{30}$ Por sua vez, $77 \%$ das mulheres com sintomatologia vasomotora apresenta dificuldades sexuais. ${ }^{3}$ Para tratamento dos sintomas vasomotores utilizam-se frequentemente antidepressivos inibidores seletivos da recaptação da serotonina; contudo, a fluoxetina e a paroxetina são contraindicadas nas doentes medicadas com tamoxifeno, pois diminuem a eficácia do tratamento oncológico. ${ }^{3}$ Por outro lado, a terapêutica hormonal de substituição sistémica também é contraindicada em doentes com antecedentes ou história atual de cancro da mama. ${ }^{11}$ Nesta população recomenda-se, por isso, utilizar preferencialmente a venlafaxina ou o citalopram, embora estes possam afetar negativamente a função sexual. ${ }^{3}$ Devem ainda ser sempre reforçadas medidas não farmacológicas de controlo dos sintomas vasomotores, como: evicção de cafeína e de álcool, diminuição da temperatura ambiente sempre que possível e utilização 
TABELA 1. As fases da resposta sexual feminina fisiológica e os principais componentes da doença e tratamento oncológicos que podem comprometer o desejo sexual, a excitação sexual e o orgasmo em mulheres com cancro da mama e cancro ginecológico

\begin{tabular}{|c|c|c|c|c|}
\hline Resposta Sexual & Desejo sexual & Excitação sexual & Orgasmo & Resolução \\
\hline $\begin{array}{l}\text { Resposta } \\
\text { fisiológica } \\
\text { feminina }\end{array}$ & $\begin{array}{l}\text { Existem várias tipologias: } \\
\text { desejo próativo ( } 15 \% \text { das } \\
\text { mulheres), responsivo } \\
(30 \%), \text { misto e ausente } \\
(6 \%) . \\
\text { Fase dependente de: } \\
\text { - Mediação androgénica; } \\
\text { - Balanço dopamina/ } \\
\text { /serotonina; } \\
\text { - Condição física/energia; } \\
\text { - Estímulo visual; } \\
\text { - Estímulo relacional } \\
\text { (componente com maior } \\
\text { impacto). }\end{array}$ & $\begin{array}{l}\text { Inclui fenómenos } \\
\text { sistémicos e locais } \\
\text { (genitais): } \\
\text { - Aumento da frequência } \\
\text { cardíaca, da tensão } \\
\text { arterial, da frequência } \\
\text { respiratória e da tensão } \\
\text { muscular; } \\
\text { - Tumescência mamária, } \\
\text { dos grandes lábios e da } \\
\text { vagina; lubrificação } \\
\text { vaginal. } \\
\text { Fase depende de: } \\
\text { - Integridade dos arcos } \\
\text { reflexos neurológicos; } \\
\text { - Vasomotricidade; } \\
\text { - Balanço } \\
\text { neurotransmissor; } \\
\text { - Testosterona (pequeno } \\
\text { contributo). }\end{array}$ & $\begin{array}{l}\text { Pode ser alcançado por } \\
\text { diferentes vias: } \\
\text { - Estímulo clitoriano } \\
\text { (90\% das mulheres); } \\
\text { - Estímulo da parede } \\
\text { anterior da vagina e colo } \\
\text { uterino (25-35\%); } \\
\text { - Fantasias sexuais; } \\
\text { - Estímulo mamário. } \\
\text { Fase depende de: } \\
\text { - Balanço } \\
\text { neurotransmissor; } \\
\text { - Integridade do sistema } \\
\text { nervoso autónomo. }\end{array}$ & $\begin{array}{l}\text { Inclui fenómenos } \\
\text { sistémicos e locais } \\
\text { (genitais): } \\
\text { - Perda de calor; } \\
\text { normalização da } \\
\text { frequência cardíaca, da } \\
\text { tensão arterial, da } \\
\text { frequência respiratória e } \\
\text { do tónus muscular; } \\
\text { - Detumescência } \\
\text { mamária, dos grandes } \\
\text { lábios e da vagina; } \\
\text { regressão clitoriana, } \\
\text { relaxamento das paredes } \\
\text { vaginais e o útero retorna } \\
\text { à posição habitual. } \\
\text { Fase depende de: } \\
\text { - Integridade dos arcos } \\
\text { reflexos neurológicos; } \\
\text { - Vasomotricidade; } \\
\text { - Balanço } \\
\text { neurotransmissor. }\end{array}$ \\
\hline $\begin{array}{l}\text { Impacto da doença } \\
\text { oncológica na } \\
\text { sexualidade }\end{array}$ & \multicolumn{4}{|c|}{$\begin{array}{l}\text { - Receção do diagnóstico - "novo eu" e "novo nós"; } \\
\text { - Progressão da doença, sintomas locais e constitucionais; } \\
\text { - Comorbilidades, nomeadamente sintomas depressivos e ansiedade. }\end{array}$} \\
\hline $\begin{array}{l}\text { Impacto do } \\
\text { tratamento } \\
\text { oncológico na } \\
\text { sexualidade }\end{array}$ & $\begin{array}{l}\text { - Redução dos } \\
\text { androgénios (cirurgia, } \\
\text { radioterapia e/ou } \\
\text { elevadas doses de } \\
\text { opioides); } \\
\text { - Alteração da } \\
\text { autoimagem (cirurgia } \\
\text { e/ou quimioterapia); } \\
\text { - Incontinência } \\
\text { esfincteriana (cirurgia } \\
\text { e/ou radioterapia); } \\
\text { - Dor; } \\
\text { - Terapêutica } \\
\text { antidepressiva } \\
\text { concomitante. }\end{array}$ & $\begin{array}{l}\text { - Diminuição da } \\
\text { lubrificação (cirurgia, } \\
\text { radioterapia, quimioterapia } \\
\text { e/ou hormonoterapia); } \\
\text { - Diminuição da } \\
\text { testosterona (cirurgia } \\
\text { e/ou radioterapia); } \\
\text { - Alteração sensitiva/zonas } \\
\text { erógenas (cirurgia, } \\
\text { radioterapia e/ou } \\
\text { quimioterapia); } \\
\text { - Lesão nervosa/vascular } \\
\text { (cirurgia e/ou radioterapia); } \\
\text { - Fibrose (radioterapia); } \\
\text { - Terapêutica } \\
\text { antidepressiva } \\
\text { concomitante. }\end{array}$ & $\begin{array}{l}\text { - Lesão nervosa (cirurgia } \\
\text { e/ou quimioterapia); } \\
\text { - Incontinência } \\
\text { esfincteriana (cirurgia } \\
\text { e/ou radioterapia) } \\
\text { - Dor; } \\
\text { - Terapêutica } \\
\text { antidepressiva } \\
\text { concomitante. }\end{array}$ & $\begin{array}{l}\text { - Lesão nervosa/ } \\
\text { /vascular (cirurgia e/ou } \\
\text { radioterapia); } \\
\text { - Fibrose (radioterapia). }\end{array}$ \\
\hline
\end{tabular}


de várias camadas de roupa, possibilitando o ajuste do vestuário às mudanças de temperatura percecionadas pela doente. ${ }^{18}$

A nível urogenital, o declínio de estradiol provocado pela hormonoterapia resulta numa redução do epitélio escamoso vulvovaginal, com diminuição do colagénio, glicogénio, mucopolisacarídeos e ácido hialorónico. ${ }^{14} \mathrm{As}-$ $\mathrm{sim}$, as paredes vaginais tornam-se mais delgadas, hiposecretórias e perdem elasticidade e, como resultado, surge secura vaginal, prurido, incontinência e dispareunia. ${ }^{3,14,26}$ Nestes casos pode ser aconselhado um lubrificante. ${ }^{3}$ Se as queixas persistirem poder-se-á recomendar lidocaína tópica previamente às relações sexuais, com alívio sintomático da dispareunia na maioria das doentes. ${ }^{3}$ Quando nenhuma das intervenções referidas é eficaz poder-se-á considerar a aplicação de estrogénio tópico (em baixas doses e com baixa absorção sistémica). ${ }^{12,19} \mathrm{O}$ estrogénio tópico estimula a proliferação do epitélio, a circulação sanguínea e as secreções glandulares locais. ${ }^{18}$ Uma revisão sistemática da Cochrane demonstrou que a aplicação local de estrogénio é a medida mais efetiva para aliviar os sintomas de atrofia vulvovaginal. ${ }^{3}$ Contudo, esta não é uma recomendação consensual nesta população, pelo que deverá ser uma decisão terapêutica ponderada do ponto de vista multidisciplinar, individualizada e partilhada com a doente., ${ }^{2,4}$

Atendendo às características histológicas e moleculares do tumor, a quimioterapia poderá ser uma opção terapêutica. ${ }^{3}$ Estima-se que $80 \%$ das doentes com cancro da mama submetidas a quimioterapia experiencie alguma alteração da função sexual nos cinco anos seguintes. ${ }^{3}$ Os seus efeitos adversos são variáveis, dependendo dos fármacos utilizados, da dose e duração do tratamento. ${ }^{3}$ De uma forma geral, o impacto da quimioterapia na sexualidade é o resultado da conjugação de três fatores: aspetos com impacto indireto na sexualidade, aspetos com impacto direto e aspetos psicológicos.

1. Aspetos físicos da quimioterapia que indiretamente podem influenciar a sexualidade:

a) Emese aguda (12-24h após tratamento), tardia (mais de 24h após tratamento) ou antecipatória. ${ }^{3}$ Como medida preventiva e terapêutica utilizam-se antagonistas do recetor 5-HT3 (como ondansetron), que não apresentam efeitos adversos a nível sexual, ao contrário dos antagonistas da do- pamina (como a metoclopramida), que devem ser evitados dado causarem hiperprolactinemia, hipogonadismo e diminuição do desejo sexual;3,36,29

b) Diarreia ou obstipação;;,26,29

c) Alopecia, com alteração importante da autoimagem; $3,18,26,29,31$

d) Mucosite que, estando associada a dor, poderá também ter impacto negativo na sexualidade da mulher $/$ casal; $^{3}$

e) Alteração da sensibilidade, que pode afetar as regiões erógenas. Nestes casos, o tratamento analgésico poderá proporcionar um alívio da sintomatologia e reduzir a sua repercussão a nível sexual. ${ }^{3}$ Os anti-inflamatórios não esteroides e o paracetamol são boas opções terapêuticas neste âmbito; já os opioides, quando tomados cronicamente, condicionam hipogonadismo, fadiga, sintomas depressivos e disfunção sexual em 90\% dos doentes; ${ }^{5}$

f) Ganho ponderal, associado às alterações da composição corporal induzidas pela quimioterapia e à fadiga. $\mathrm{O}$ aumento de peso pode afetar negativamente a autoestima, aumentar o risco cardiovascular e o risco de diabetes (que, por sua vez, também afetam negativamente a sexualidade);,3,5

g) Chemobrain, caracterizado por declínio cognitivo, falhas mnésicas, alteração da concentração e da memória executiva. ${ }^{18}$

2. Aspetos físicos da quimioterapia que influenciam diretamente a sexualidade e fertilidade: menor lubrificação vaginal, dispareunia, alteração do funcionamento nervoso autónomo e menopausa iatrogénica. ${ }^{3,18,26-27}$

Relativamente à fertilidade, tanto a quimioterapia como a hormonoterapia poderão ter um efeito negativo. ${ }^{32}$ A quimioterapia reduz a fertilidade diretamente (temporária ou permanentemente), dado induzir a falência ovárica. Por outro lado, a hormonoterapia afeta indiretamente a fertilidade, uma vez que induz toxicidade fetal, sendo desaconselhada a gravidez durante $o$ tratamento. ${ }^{32}$ Neste âmbito, todas as doentes com neoplasia em idade jovem deverão ser precocemente informadas sobre o possível impacto negativo do tratamento na fertilidade, assim como sobre a possibilidade de recorrer a técnicas de preservação da fertilidade, de forma a minorar o impacto dos tratamentos numa 
possível gravidez futura. ${ }^{17,33}$ Caso a doente se apresente interessada deverá ser referenciada, quanto antes, para um centro especializado em técnicas de preservação da fertilidade (previamente ao início do tratamento oncológico)..$^{17,34} \mathrm{~A}$ referenciação precoce permite a avaliação multidisciplinar e planeamento atempado, a fim de determinar qual o método de preservação da fertilidade que melhor se adequa à situação clínica, à idade da doente, às suas preferências, à urgência do tratamento, à presença de parceiro e aos recursos locais. ${ }^{31,35-36}$ Os métodos de preservação da fertilidade mais utilizados consistem na criopreservação de ovócitos fecundados e ovócitos maduros. Estas técnicas requerem estimulação ovárica, o que atrasa o início da quimioterapia entre duas a cinco semanas, não sendo recomendado para doentes que não possam adiar o início do tratamento oncológico. ${ }^{34}$ Existem outros métodos, menos difundidos na prática clínica, que não requerem estimulação ovárica, nomeadamente a criopreservação do tecido ovárico, a criopreservação de ovócitos imaturos e, menos consensual, a utilização de análogos da GnRH durante os tratamentos de quimioterapia (que reduz a toxicidade do tratamento ao nível dos ovários, aumentando a probabilidade de conceção futura). ${ }^{3,34-35}$

3. Aspetos psicológicos associados à quimioterapia: salientam-se as crenças disfuncionais sobre o cancro, sentimentos de culpa, depressão, reações negativas face à modificação da imagem corporal e disfunção nas relações interpessoais. ${ }^{3,5,26-27,31-32,37-38}$

\section{Cancro ginecológico}

As neoplasias ginecológicas incluem: o cancro do ovário (36\% dos tumores malignos ginecológicos), o cancro do endométrio (36\%), o cancro do útero (23\%), o cancro da vulva ( 3 a $4 \%$ ) e o cancro da vagina (1 a $2 \%)^{3,39}$

\section{Neoplasia do ovário}

O cancro do ovário constitui 3\% do total de neoplasias em mulheres. ${ }^{3}$ Contudo, é responsável por mais mortes do que qualquer outra neoplasia do sistema reprodutor. $^{3}$

A maioria das doentes com cancro ovárico (57\%) apresenta sintomas adversos do tratamento. ${ }^{40} \mathrm{~A}$ linfadenectomia retroperitoneal radical pode lesar os ner- vos do sistema autónomo. ${ }^{3}$ Por outro lado, a cirurgia em mulheres em idade fértil resulta em menopausa iatrogénica, condicionando sintomas vasomotores, alterações do humor e do sono com impacto na qualidade de vida e no desejo sexual. ${ }^{3}$

É de salientar que a maioria das doentes com cancro ovárico considera a sua saúde sexual importante. ${ }^{29,40-41}$ Contudo, apresenta menor prazer sexual e mais desconforto nas relações sexuais. ${ }^{22}$

\section{Neoplasia do endométrio}

O cancro do endométrio apresenta taxas de sobrevivência de 72 a $84 \%$ a cinco anos. ${ }^{2}$ A maioria das doentes é diagnosticada num estádio precoce, para o qual a cirurgia é o tratamento de eleição. ${ }^{2}$ Atualmente, a intervenção cirúrgica é realizada por via laparoscópica, permitindo menor tempo de hospitalização, uma recuperação mais rápida e menor morbilidade. ${ }^{2}$

No entanto, estima-se que $89 \%$ das doentes com estádios iniciais de cancro do endométrio apresente disfunção sexual, sendo o principal sintoma referido a dor, seguida da diminuição da lubrificação, diminuição do desejo e dificuldade no atingimento do orgasmo. ${ }^{43}$ Os principais efeitos adversos da terapêutica do cancro do endométrio na sexualidade advêm da radioterapia.. ${ }^{43-44}$ A interação da radiação ionizante com os tecidos ativa citocinas e liberta fatores de crescimento que conduzem à fibrose. ${ }^{45-46} \mathrm{~A}$ fibrose traduz-se em secura vaginal, dispareunia, encurtamento e estenose vaginais, associando-se a um declínio da qualidade de vida global.5,46 Neste contexto, estima-se que $63 \%$ das doentes sexualmente ativas previamente ao diagnóstico permaneça sexualmente ativa após o tratamento, apresentando, contudo, uma redução da frequência das relações sexuais..$^{48} \mathrm{O}$ encurtamento vaginal, mais especificamente, é relatado por metade das doentes submetidas a radioterapia, sendo que $45 \%$ referem que nunca, ou apenas ocasionalmente, conseguem completar a relação sexual. ${ }^{48} \mathrm{~A}$ braquiterapia diminuiu o risco de efeitos adversos da radioterapia convencional, estando associada a menor limitação nas atividades diárias. ${ }^{3}$ No entanto, a braquiterapia também não é isenta de efeitos adversos, podendo associar-se a alterações da mucosa vaginal, que resultam em secura, dispareunia e fibrose, com impacto nefasto na sexualidade. ${ }^{3,47}$ Para tratamento da estenose e encurtamento vaginal existem 
programas de reabilitação realizados por fisiatras e equipas de enfermagem especializadas, que utilizam dilatadores vaginais, estrogénios locais e lubrificantes. ${ }^{49-50}$ Existem dilatadores rígidos e maleáveis, disponíveis em vários tamanhos..$^{45} \mathrm{~A}$ expansão tecidual vaginal estimulará a mitose e o desenvolvimento de novas células epiteliais. ${ }^{51} \mathrm{~A}$ intervenção precoce é importante, pois o hipoestrogenismo leva a atrofia vaginal crónica. ${ }^{45} \mathrm{~A}$ duração e frequência da utilização dos dilatadores pode variar de três minutos de utilização, duas vezes por semana, até dez minutos de utilização, duas vezes por dia durante seis meses. ${ }^{45,49,52}$ Posteriormente, começar-se-á a espaçar as utilizações: habitualmente com uma utilização semanal durante um ano ${ }^{45,49,52} \mathrm{Du}-$ rante este processo dever-se-á avaliar com regularidade a necessidade de manutenção do programa de reabilitação; por exemplo, uma doente sexualmente ativa ou assintomática à observação ginecológica poderá suspender o seu programa reabilitativo. ${ }^{49,52}$

Em resumo, o tratamento do cancro do endométrio apresenta muitos desafios. As doentes submetidas a cirurgia apresentam elevadas taxas de disfunção sexual; já as doentes submetidas quer a cirurgia como a radioterapia apresentam elevada morbilidade e uma diminuição significativa da satisfação sexual global se não forem incluídas em programas de reabilitação. ${ }^{18}$

\section{Neoplasia do útero}

O cancro do útero localizado no corpo uterino é a sexta neoplasia com maior incidência na população feminina a nível mundial; já o cancro do colo do útero corresponde à quarta doença oncológica mais frequente nesta população..$^{53}$

A histerectomia radical é uma cirurgia (física e emocionalmente) agressiva. ${ }^{18} \mathrm{~A}$ histerectomia radical inclui a resseção de nervos e do suprimento vascular para a vagina. ${ }^{40}$ Os efeitos secundários deste procedimento a nível sexual são: diminuição de líbido (25-57\%), diminuição da sensibilidade dos lábios vaginais $(71 \%)$, diminuição da lubrificação vaginal (10-26\%), encurtamento vaginal (25\%) e dispareunia (18\%). ${ }^{40} \mathrm{~A}$ histerectomia radical pode, ainda, causar lesão dos plexos nervosos hipogástrico e esplâncnico, conduzindo a alterações da função vesical e intestinal. ${ }^{3}$ As técnicas de nerve-sparing modificadas têm sido propostas para diminuir a morbilidade associada à lesão vasculo-nervo- sa. $^{3}$ Adicionalmente, qualquer histerectomia causa infertilidade, pelo que a fertilidade deve ser um aspeto debatido antes do tratamento. ${ }^{2}$ A deteção precoce deste tipo de neoplasias permite evitar a morbilidade associada à intervenção cirúrgica, através da realização de técnicas menos invasivas, como a conização, que poderão permitir a manutenção da fertilidade. ${ }^{40}$

Por outro lado, a radioterapia no contexto de cancro do útero pode levar à disfunção vesical e intestinal. A incontinência de esfíncteres pode apresentar um impacto negativo na autoestima e associa-se ao receio de perdas involuntárias durante o ato sexual. ${ }^{2,48,54} \mathrm{O}$ linfedema dos membros inferiores (resultante da linfadenectomia) também é um efeito adverso mais acentuado em doentes sob radioterapia. ${ }^{2}$

Salienta-se que a infeção pelo papillomavírus humano (HPV) é uma doença sexualmente transmissível, causalmente associada ao aparecimento de neoplasia do colo do útero. Para muitas doentes o diagnóstico de cancro do colo do útero associa-se, por isso, a estigma social e poderá ter um impacto negativo no casal. Os profissionais de saúde devem estar particularmente atentos, de forma a evitar que este seja um obstáculo ao rastreio, diagnóstico e tratamento precoces. ${ }^{2}$

Em resumo, as doentes com cancro do útero apresentam maior disfunção sexual se submetidas a cirurgia radial e radioterapia. As disfunções vesical e intestinal impactam negativamente a vida íntima, podendo ser diminuídas com a técnica de nerve-sparing. ${ }^{3}$ Nos casos de dispareunia deve intervir-se precocemente com lubrificantes e dar ênfase a diferentes formas de estimulação sexual e de relaxamento vaginal. ${ }^{3}$

\section{Neoplasia da vulva}

O cancro vulvar é mais comum em mulheres idosas, mas nos últimos anos tem-se verificado um aumento da incidência nas camadas populacionais jovens, associado ao HPV. ${ }^{2}$ No passado o tratamento consistia na vulvectomia radical (com remoção de todos os genitais externos, incluindo clitóris, pequenos e grandes lábios e linfadenectomia). ${ }^{3}$ Atualmente são raras as cirurgias radicais. ${ }^{2-3}$ Contudo, por vezes, a necessidade de obtenção de margens negativas pode levar à exérese do clítoris, com compromisso importante do prazer sexual. ${ }^{2-3}$

A medicina reconstrutiva é de particular importância para a saúde sexual da mulher com este tipo de 
neoplasia, permitindo a reconstrução dos genitais externos com retalhos locais ou oriundos de outras regiões anatómicas (como a coxa)., ${ }^{3,15}$ Esta mudança de paradigma possibilita a muitas doentes voltarem a ser sexualmente ativas, bem como manterem a vigilância ginecológica aconselhada, pois a reconstrução da vulva e do canal vaginal permite manter a realização de citologia para rastreio do cancro do colo uterino., ${ }^{3,15}$

Em resumo, a cirurgia vulvar tem impacto negativo na sexualidade das doentes com cancro da vulva. ${ }^{3}$ Nos casos em que se verifica uma hipostesia marcada dever-se-á recomendar utilização de lubrificantes. ${ }^{5}$ Esta medida pode ajudar a recuperar parte da sensibilidade e facilitar o ato sexual. Em situações particulares é necessária a intervenção reconstrutiva; no entanto, o compromisso sensitivo estará muito frequentemente presente. $^{5}$

\section{Neoplasia da vagina}

A neoplasia primária da vagina é uma patologia pouco comum e heterogénea, apresentando um pico de incidência nas sexta e sétima décadas de vida. ${ }^{55-57}$ As doentes com antecedentes de outros tipos de cancro ginecológico apresentam um risco aumentado de desenvolver cancro da vagina, dada a proximidade anatómica e exposição a fatores carcinogénicos comuns, como a infeção por HPV. ${ }^{55-56,58}$ A doença poderá interferir com a sexualidade da doente quando associada a hemorragia vaginal ou dor pélvica. .6,59 $^{5,5}$

O tratamento habitualmente é composto por radioterapia e/ou cirurgia, associada a técnicas reconstrutivas. ${ }^{56-57}$ Neste contexto, a doente deverá ser informada acerca da previsível alteração de sensibilidade da neovagina e sua implicação no prazer sexual. ${ }^{58} \mathrm{~A}$ aplicação de estrogénio tópico poderá melhorar a vascularização e elasticidade dos tecidos. ${ }^{58} \mathrm{~A}$ satisfação sexual e o orgasmo são possíveis com enxertos vaginais e as doentes deverão ser encorajadas a permanecer sexualmente ativas após a cicatrização cirúrgica. ${ }^{58}$ Quanto à radioterapia, as suas consequências na sexualidade são semelhantes aos efeitos do tratamento da neoplasia do endométrio, previamente descritos.

\section{Situações específicas}

\section{Menopausa iatrogénica}

A disfunção ovárica precoce tem inúmeras etiolo- gias, podendo ser causada por alterações genéticas, doenças autoimunes, doenças infeciosas ou por iatrogenia (que compreende a menopausa secundária a muitos dos tratamentos oncológicos supradescritos). ${ }^{60}$ A causa mais frequente de menopausa iatrogénica é a ooferectomia bilateral (correspondendo a $25 \%$ dos casos). ${ }^{55,60}$

Na menopausa iatrogénica ocorre uma diminuição abrupta do estrogénio, progesterona e testosterona, desencadeando sintomas que (apesar de serem sintomas comuns aos de várias mulheres após a falência ovárica fisiológica), tendo um início abrupto, poderão ser bastante intensos e apresentar grandes implicações na qualidade de vida. ${ }^{3,60}$ A redução dos estrogénios tem como consequências a atrofia vaginal, perda da elasticidade dos tecidos e a diminuição da lubrificação vaginal. ${ }^{3,54,60}$ Estas alterações poderão condicionar dispareunia e prejudicar o orgasmo. ${ }^{3,55}$ Verifica-se ainda uma redução da testosterona, que condiciona a diminuição do desejo sexual. ${ }^{3,54}$ Nestes casos pode ser equacionada a terapêutica hormonal de substituição, na ausência de contraindicações. ${ }^{40}$ Após uma histerectomia, a terapêutica hormonal de substituição pode fazer-se apenas com estrogénios; já nas doentes que mantêm útero dever-se-á adotar uma terapêutica combinada também com progestagénios, evitando a proliferação endometrial. ${ }^{5,55} \mathrm{~A}$ via transdérmica deverá ser usada sempre que possível para bypass à metabolização hepática e para evitar fenómenos tromboembólicos.,55 Também existem estudos que relatam uma melhoria do desejo sexual em doentes sob terapêutica com testosterona. ${ }^{55}$ No entanto, os dados acerca da sua segurança e eficácia a longo prazo são limitados, pelo que a sua utilização não é recomendada. ${ }^{55,61}$

Adicionalmente, a menopausa iatrogénica apresenta também aspetos psicológicos e sociais importantes para os quais contribuem a idade da doente, a fase do ciclo de vida em que se encontra e fatores socioculturais. ${ }^{54,60}$ Esta população reporta pior estado de saúde global, menor nível de bem-estar psicológico, maior prevalência de dor crónica, menor satisfação sexual e pior qualidade de vida. ${ }^{36}$

\section{Comorbilidades psicológicas}

Os fármacos psicotrópicos são utilizados em $79 \%$ dos doentes oncológicos no contexto de ansiedade e/ou 
depressão. ${ }^{5}$ Para além destas indicações, são por vezes utilizados, mesmo em baixas doses, para alívio da dor neuropática, melhoria dos sintomas vasomotores e da qualidade do sono. ${ }^{5}$ Contudo, a maioria dos antidepressivos pode afetar negativamente a função sexual e agravar distúrbios sexuais pré-existentes. ${ }^{8,62}$ Perante a apresentação de sintomas de disfunção sexual em doentes medicados com estes fármacos dever-se-á optar por outros fármacos antidepressivos ou hipnóticos, com efeito neutro ou positivo sobre a função sexual, nomeadamente o bupropion, a mirtazapina e a trazodona. ${ }^{8,62}$

\section{Abordagem da sexologia pelo profissional de saúde} Como abordado previamente, existe uma elevada prevalência de disfunção sexual nas doentes com cancro da mama e cancro ginecológico, mas a sexualidade é um tópico pouco abordado pelos médicos que acompanham esta população. Paralelamente, nos casos em que os profissionais de saúde abordam a temática da sexualidade no doente oncológico, verifica-se uma melhoria da relação médico-doente, um fortalecimento da aliança terapêutica e uma melhoria da adesão da utente ao plano de cuidados.,12

Uma das características da medicina geral e familiar é a prestação de cuidados longitudinais. $\mathrm{O}$ médico de família poderá acompanhar as utentes com cancro da mama e cancro ginecológico desde o momento do diagnóstico, passando pelo período de tratamento, pela reabilitação e, por vezes, pelas fases paliativa e terminal. ${ }^{3}$ Durante a prestação de cuidados, o médico deverá abordar regularmente a sexualidade, de forma integrada na entrevista clínica (a título de exemplo, questionar as alterações na sexualidade depois de avaliar a fadiga e as alterações de humor). ${ }^{3}$ Para facilitar a introdução do tema da sexualidade na comunicação existem modelos práticos, nomeadamente o Modelo dos 5 A's, o PLISSIT e o Brief Sexuality Related Communication.

O Modelo dos 5 A's foi inicialmente descrito no contexto da intervenção breve em doentes fumadores motivados para deixar de fumar. ${ }^{63-64}$ Este método é amplamente utilizado nos cuidados de saúde primários e visa avaliar sistematicamente os hábitos tabágicos dos utentes, de forma oportunista. ${ }^{63} \mathrm{O}$ Modelo dos 5 A's poderá ser adaptado para a avaliação da sexualidade das doentes com cancro da mama e cancro ginecológico, facili- tando a comunicação com as mesmas, promovendo a partilha dos problemas sexuais que experienciam e a exploração dos fatores biomédicos, psicológicos e relacionais que contribuem para a manutenção da disfunção vivenciada. O modelo engloba os seguintes passos: ${ }^{3,40,65}$

- Abordar. Dever-se-á garantir a privacidade e confidencialidade, adotar uma postura profissional, não emitir juízos de valor e respeitar a doente e o casal, tendo em conta possíveis diferenças socioculturais. ${ }^{3}$ O médico deverá começar por questionar possíveis alterações na vida íntima secundárias ao diagnóstico da doença oncológica, privilegiando perguntas abertas. ${ }^{3}$ Por vezes, os profissionais de saúde optam por iniciar o tema com questões acerca dos efeitos adversos do tratamento. ${ }^{3} \mathrm{Um}$ dos exemplos como o tema da sexualidade poderá ser introduzido na prática clínica é do seguinte modo: "Quando um doente é diagnosticado com cancro $\mathrm{x}$ (ou faz o tratamento y), normalmente discutimos as suas preocupações a nível sexual. Como é que o diagnóstico (ou o tratamento) afetou a forma como se vê a si mesma?" Quais são os aspetos da sexualidade que foram mais afetados com a doença?" ${ }^{44,65}$

- Aconselhar. A doente deve ter acesso a informação e ver esclarecidas as suas dúvidas. ${ }^{3,66}$ Deverá ser informada acerca do possível impacto da doença oncológica e respetivo tratamento na sexualidade, de forma a conseguir preparar-se e adotar estratégias adaptativas. ${ }^{3}$ Deve salientar-se a grande variabilidade individual do impacto do tratamento oncológico na sexualidade. ${ }^{3}$

- Avaliar. Este passo visa a realização da história do problema sexual, que inclui a compreensão do funcionamento sexual prévio ao diagnóstico, dificuldades atuais e formulação de um plano terapêutico. ${ }^{7}$ Privilegiam-se questões interligadas para determinar o tipo e grau de disfunção apresentada, as causas e os fatores promotores da sua manutenção. ${ }^{7}$

- Apoiar. O plano terapêutico deve ser personalizado e realizado em aliança terapêutica com a doente (e $\mathrm{o} / \mathrm{a}$ parceiro/a), procurando também a psicoeducação em sexualidade: encorajar comunicação e reflexão sobre a vida sexual antes e depois do tratamento; promover a discussão das preocupações da doente; capacitar a doente a lidar com as alterações físi- 
cas; introduzir técnicas de relaxamento e formas de potenciar ou recriar prazer; encarar a nova fase como uma forma de autodescoberta sensitiva e reforçar positivamente a reinvenção da vida sexual após o cancro. $^{8,14}$

- Acompanhar. Inclui o seguimento com revisão do plano terapêutico e de eventuais dificuldades en-

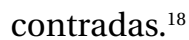

Outra das ferramentas utilizadas é o PLISSIT que consiste num modelo de rastreio de patologia sexual em consulta, constituído por quatro níveis de intervenção: ${ }^{24,67}$

P-Permissão (Permission). Colocando uma questão como "Será que podemos falar sobre sexualidade?", o médico demonstra disponibilidade para ouvir as preocupações da doente acerca deste tema, comunicando interesse e respeito. Na maioria das vezes a doente apenas pretende ver validados os seus pensamentos, sentimentos e comportamentos, bem como ter a perceção de que os seus receios e dúvidas são comuns e legítimos. ${ }^{67}$

LI - Informação Limitada (Limited information). Fase que poderá ser introduzida por uma questão como: "Algumas pessoas com cancro da mama/cancro ginecológico referem alguns problemas a nível sexual. Isto acontece consigo?" O objetivo desta fase é compreender a dúvida/receio/preocupação da doente à qual se pretende responder com informação concisa, clara e relevante. Muitas vezes surge logo a seguir ao pedido de permissão inicial. ${ }^{67}$

SS - Sugestões Específicas (Specific suggestions). Esta fase é precedida da colheita da história do problema sexual que deverá incluir a descrição do problema, a data de início, os eventos precipitantes, a evolução, a frequência e a intensidade dos sintomas, os fatores moduladores, o impacto funcional, as crenças e ideias associadas, bem como informação sobre as avaliações médicas anteriores, existência de tratamentos prévios e as expectativas da doente, de acordo com objetivos realistas. Posteriormente à colheita de informação, o médico fornece sugestões específicas que se adequam ao problema particular da doente (ou do casal). ${ }^{67}$

$\mathrm{T}$-Terapia Intensiva (Intensive therapy). Consiste na abordagem terapêutica comportamental especializada e individualizada para as doentes que não res- ponderam às fases anteriores. Raramente é necessária, dado que $70 \%$ dos problemas são resolvidos no primeiro nível de intervenção e 80-90\% são resolvidos ao longo dos três primeiros níveis visados pelo PLISSIT. $^{24,67}$

Existem ainda outros modelos, como o ALARM, descrito por Andersen em 1990, que visa os passos: Activity, Libido, Arousal, Resolution, Medical information; e o BETTER, descrito por Cohen em 2004, que consiste nos seguintes: Bringing up the topic of sexuality, Explaining, Telling, Timing, Recording. ${ }^{24}$ Mais recentemente, em 2015, a Organização Mundial da Saúde desenvolveu a ferramenta Brief Sexuality Related Communication (BSRC), que privilegia perguntas abertas, organizadas do seguinte modo:

- Abordar. Estabelecimento da relação médico-doente e a utilização de perguntas-tipo para introduzir o tema da sexualidade ("Há alguma dúvida relacionada com assuntos do foro sexual?"); ${ }^{12}$

- Responder. Utilização de questões abertas acerca da sexualidade que permitam abrir a comunicação (“Está satisfeito com a sua vida sexual?");12

- Personalizar. Identificação próxima com os problemas, dificuldades ou doenças da utente ("Algumas pessoas com cancro da mama/cancro ginecológico têm alguns problemas a nível sexual. Como é isto para si?"); ${ }^{12}$

- Iniciar o aconselhamento. Fornecer informação adequada e identificar as diferentes fases do plano terapêutico. ${ }^{12}$

Por fim, outra forma de avaliar a sexualidade regularmente poderá constar na utilização de questionários, nomeadamente o Brief Sexual Symptom Checklist: Women's version ou, mais especificamente, a versão portuguesa do Sexual Activity Questionnaire, validada para avaliação da função sexual em doentes com cancro da mama. ${ }^{3}$

Em resumo, existem várias formas que o médico poderá adotar na abordagem da sexualidade da doente oncológica, cabendo-lhe optar por aquela que lhe for mais conveniente, de acordo com o seu local de trabalho, população-alvo, tempo disponível, aptidão e preferência. ${ }^{67}$ Qualquer que seja o método utilizado, no final da entrevista clínica, o médico deverá ser capaz de identificar o problema sexual experienciado pela doente numa perspetiva biopsicossocial; compreender o 
impacto de problemas sexuais preexistentes e quais os recursos da doente/do casal; analisar o impacto da doença oncológica e do respetivo tratamento na sexualidade e fertilidade. ${ }^{3}$ Não se procura resolver todos os problemas sexuais dos doentes, mas identificar e gerir a maioria das situações. ${ }^{67}$

\section{Reflexão sobre o papel do médico de família}

O médico de família encontra-se numa posição privilegiada na gestão dos problemas sexuais apresentados pelas utentes com cancro da mama ou cancro ginecológico. Por um lado, beneficia da relação médico-doente (muitas vezes já estabelecida previamente à data do diagnóstico da doença oncológica); por outro lado, conhece os antecedentes pessoais, familiares e socioculturais da utente, a fase do ciclo de vida em que se encontra, a dinâmica relacional do casal e da família, bem como qual a rede de apoio existente. Adicionalmente, tem a possibilidade de acompanhar a doente ao longo do tempo, prestando cuidados longitudinais e holísticos. O médico de família poderá, por isso, explorar os conceitos de doença e dolência, bem como compreender o impacto da doença oncológica e seu tratamento nos diferentes fatores influenciadores da função sexual - físico (função orgânica), psíquico (autoestima e imagem corporal), existencial (significado da vida, valores e amor), interpessoal (relação com parceiro/a, filhos e amigos), sociocultural (política e religião) e sexual (atração e desejo).

Nesta interação cabe ao médico ceder informação de forma progressiva e de acordo com a vontade da doente e pesquisar motivos latentes de vinda à consulta, que podem consistir em crenças, receios e preocupações sobre a sexualidade. Deverá ser implementada uma estratégia de reabilitação holística da mulher com neoplasia mamária ou ginecológica, promovendo mudanças de estilo de vida, como a atividade física regular (prevenindo a sarcopenia, reduzindo a depressão e melhorando a qualidade do sono), uma alimentação equilibrada e controlo do peso corporal (que melhora a autoimagem, melhora o humor, reduz a fadiga e as queixas álgicas), a cessação tabágica, a evicção de bebidas alcoólicas e a higiene do sono. Deverá, ainda, ser realizada uma revisão da medicação a cada consulta e avaliadas situações que podem influenciar negativamente a sexualidade, como a secura vaginal, a dor crónica, insónia e fadiga. .,54 $^{3,5}$
Quando é identificada qualquer disfunção sexual, o médico de família que acompanha a doente poderá propor um plano terapêutico específico para a situação identificada e/ou ponderar a referenciação, caso seja necessário apoio especializado. ${ }^{3}$ Poderá ser necessária a intervenção de diferentes profissionais de saúde em diferentes momentos da doença, nomeadamente apoio por parte de ginecologia, oncologia, medicina interna (para estudo de doenças crónicas com repercussões na sexualidade), psicologia (se existirem problemas pessoais inibitórios da função sexual), psiquiatria (perante sintomas depressivos ou ansiedade não responsivos à primeira linha de tratamento ou cuja terapêutica apresenta efeitos adversos de difícil gestão nos cuidados de saúde primários), urologia/uro-ginecologia e medicina física e reabilitativa (e.g., perante patologia do pavimento pélvico). ${ }^{11,17,54}$ Poderá ainda ser equacionada a terapia de casal, quando os problemas de relacionamento do casal são os principais contribuintes para a disfunção sexual, e/ou a avaliação por sexologia clínica, nomeadamente perante dificuldade no atingimento do orgasmo ou perda da motivação sexual em qualquer um dos parceiros. ${ }^{11,17,54}$

Ao longo do seguimento médico é importante conhecer e atualizar as expectativas da utente, indo ao seu encontro ou reformulando-as, de acordo com os recursos disponíveis. Espera-se do médico de família uma atitude integrativa e pró-ativa em relação à sexualidade, envolvendo a doente no plano de cuidados.

\section{CONCLUSÃO}

A presente revisão narrativa teve como objetivo descrever os problemas sexuais das mulheres com cancro da mama e cancro ginecológico. Identificaram-se ainda algumas estratégias para controlo dos sintomas associados à disfunção sexual nesta população e foram descritas técnicas comunicacionais facilitadoras da abordagem à sexualidade.

A maioria das mulheres com cancro da mama ou cancro ginecológico apresenta problemas sexuais. No caso da neoplasia da mama a cirurgia poderá alterar a autoimagem, a sensibilidade e a mobilidade da doente, afetando principalmente os domínios do desejo e excitação sexual. A radioterapia poderá condicionar alterações sensitivas, fibrose tecidular, insónia e fadiga, potencialmente afetando o desejo e excitação sexual. A 
hormonoterapia associa-se a sintomas de privação hormonal que afetam o desejo, excitação sexual e orgasmo. Dado que também a terapêutica antidepressiva pode afetar negativamente a função sexual, privilegiam-se medidas não farmacológicas para alívio dos sintomas vasomotores, assim como terapêutica tópica não hormonal para o tratamento dos sintomas urogenitais. Quanto à quimioterapia, verificam-se múltiplos efeitos que direta ou indiretamente prejudicam todas as fases da resposta sexual, como a alteração da autoimagem, a diminuição da lubrificação vaginal, a alteração do sistema nervoso autónomo e a menopausa iatrogénica.

No caso das neoplasias ginecológicas, as patologias mais frequentes são o cancro do ovário, do endométrio e do útero. A cirurgia da neoplasia do ovário poderá implicar a menopausa iatrogénica, lesão nervosa e diminuição da lubrificação vaginal, afetando todas as fases da resposta sexual. Por outro lado, os principais efeitos adversos do tratamento da neoplasia do endométrio advêm da radioterapia, que pode causar estenose vaginal, salientando-se a importância da integração da doente num programa de reabilitação. No âmbito da neoplasia uterina, a histerectomia também pode alterar todas as fases da resposta sexual feminina, dado o risco de lesão vasculo-nervosa, afeção sensitiva, diminuição da lubrificação vaginal e infertilidade. A deteção precoce deste tipo de neoplasias permite evitar a morbilidade deste procedimento, através da utilização de técnicas menos invasivas.

Constata-se, assim, que a maioria dos tratamentos no contexto do cancro da mama e cancro ginecológico têm um impacto negativo na sexualidade e que todas as fases da resposta sexual podem ser afetadas, em conjunto ou separadamente, de forma variável, de acordo com a terapêutica adotada.

Neste âmbito, o médico é responsável por salvaguardar a permissão para abordar a sexualidade, fornecer informação sobre o impacto da neoplasia e respetivo tratamento nesta área e procurar soluções adaptadas à doença e à dolência de cada utente. Existem questionários e modelos facilitadores da comunicação, cabendo ao médico optar pela ferramenta que melhor se adapta à sua prática e a cada caso em particular. No futuro seria importante uma maior consciencialização, formação e treino dos profissionais de saúde que acompanham estas doentes na abordagem da temática da se- xualidade, nomeadamente através da promoção de ações de formação sobre a intervenção breve na área da sexualidade $^{12}$ (habitualmente pouco aprofundada no ensino pré-graduado), bem como com a introdução dos modelos e questionários validados nas plataformas informáticas de apoio à consulta.

\section{REFERÊNCIAS BIBLIOGRÁFICAS}

1. World Health Organization. Sexual health and its linkages to reproductive health: an operational approach. Geneva: WHO; 2017. ISBN 978924151288

2. Kennedy V, Abramsohn E, Makelarski J, Barber R, Wroblewski K, Tenney $M$, et al. Can you ask? We just did! Assessing sexual function and concerns in patients presenting for initial gynecologic oncology consultation. Gynecol Oncol. 2015;137(1):119-24.

3. Reisman Y, Gianotten W. Cancer, intimacy and sexuality: a practical approach. Springer International Publishing; 2017. ISBN 9783319431932

4. Krychman ML. Sexual rehabilitation medicine in a female oncology setting. Gynecol Oncol. 2006;101(3):380-4.

5. Krebs LU. Sexual assessment in cancer care: concepts, methods, and strategies for success. Semin Oncol Nurs. 2008;24(2):80-90.

6. Schover LR, McKee AL. Sexuality rehabilitation. Rehabil Oncol. 2000; 18(1):16-8.

7. Perz J, Ussher JM, Gilbert E. Constructions of sex and intimacy after cancer: Q methodology study of people with cancer, their partners, and health professionals. BMC Cancer. 2013;13:270.

8. Enzlin P, De Clippeleir I. The emerging field of 'oncosexology': recognising the importance of addressing sexuality in oncology. Belg J Med Oncol. 2011;5(2):44-9.

9. Krebs L. What should I say? Talking with patients about sexuality issues. Clin J Oncol Nurs. 2006;10(3):313-5.

10. Stabile C, Goldfarb S, Baser RE, Goldfrank DJ, Abu-Rustum NR, Barakat RR, et al. Sexual health needs and educational intervention preferences for women with cancer. Breast Cancer Res Treat. 2017;165(1):7784.

11. Carter J, Lacchetti C, Andersen BL, Barton DL, Bolte S, Damast S, et al. Interventions to address sexual problems in people with cancer:American Society of Clinical Oncology clinical practice guideline adaptation of cancer care Ontario guideline. J Clin Oncol. 2018;36(5):492511.

12. World Health Organization. Brief sexuality-related communication: recommendations for a public health approach. Geneva: WHO; 2015. ISBN 9789241549004

13. Flynn KE, Reese JB, Jeffery DD, Abernethy AP, Lin L, Shelby RA, et al. Patient experiences with communication about sex during and after treatment for cancer. Psychooncology. 2012;21(6):594-601.

14. Costa FA, Ribeiro MC, Braga S, Carvalho E, Francisco F, Miranda AC, et al. Sexual dysfunction in breast cancer survivors: cross-cultural adaptation of the sexual activity questionnaire for use in Portugal.Acta Med Port. 2016;29(9):533-41.

15. Barros F, Figueiredo R. Manual de medicina sexual: visão multidisciplinar. HSJ Consultores; 2014.

16. DeSantis CE, Lin CC, Mariotto AB, Siegel RL, Stein KD, Kramer JL, et al. 
Cancer treatment and survivorship statistics, 2014. CA Cancer J Clin. 2014;64(4):252-71.

17. Paluch-Shimon S, Pagani O, Partridge AH, Abulkhair O, Cardoso MJ, Dent $\mathrm{RA}$, et al. ESO-ESMO 3rd international nconsensus guidelines for breast cancer in young women (BCY3). Breast. 2017;35:203-17.

18. Krychman ML, Pereira L, Carter J, Amsterdam A. Sexual oncology: sexual health issues in women with cancer. Oncology. 2006;71(1-2):1825.

19. Fosså SD, Dahl AA. Fertility and sexuality in young cancer survivors who have adult-onset malignancies. Hematol Oncol Clin North Am. 2008;22(2):291-303.

20. Georgiadis JR, Kringelbach ML. The human sexual response cycle: brain imaging evidence linking sex to other pleasures. Prog Neurobiol. 2012;98(1):49-81.

21. Rosen RC, Barsky JL. Normal sexual response in women. Obstet Gynecol Clin North Am. 2006;33(4):515-26.

22. Maiorino Ml, Chiodini P, Bellastella G, Giugliano D, Esposito K. Sexual dysfunction in women with cancer: a systematic review with metaanalysis of studies using the Female Sexual Function Index. Endocrine. 2016;54(2):329-41.

23. Cavalheiro JA, Bittelbrunn AC, Menke CH, Biazús JV, Xavier NL, Cericatto $R$, et al. Sexual function and chemotherapy in postmenopausal women with breast cancer. BMC Womens Health. 2012;12:28.

24. Faghani S, Ghaffari F. Effects of sexual rehabilitation using the PLISSIT model on quality of sexual life and sexual functioning in post-mastectomy breast cancer survivors. Asian Pac J Cancer Prev. 2016;17(11) 4845-51.

25. Archangelo SC, Neto MS, Veiga DF, Garcia EB, Ferreira LM. Sexuality, depression and body image after breast reconstruction. Clinics. 2019;74:e883.

26. KolodziejczykA, Pawlowski T. Negative body image in breast cancer patients. Adv Clin Exp Med. 2019;28(8):1137-42.

27. Pinto AC. Sexuality and breast cancer: prime time for young patients. J Thorac Dis. 2013;5(Suppl 1):S81-6.

28. Streb J, Jabło ski MJ, Stowik A, Babczyk D, Jach R. Indications for sexology consultation in women after surgical treatment due to breast cancer. Ann Agric Environ Med. 2019;26(2):379-84.

29. Dizon DS, Suzin D, Mcllvenna S. Sexual health as a survivorship issue for female cancer survivors. Oncologist. 2014;19(2):202-10.

30. Holli K, Valavaara R, Blanco G, Kataja V, Hietanen P, Flander M, et al. Safety and efficacy results of a randomized trial comparing adjuvant toremifene and tamoxifen in postmenopausal patients with node-positive breast cancer. J Clin Oncol. 2000;18(20):3487-94.

31. Reese JB, Keefe FJ, Somers TJ, Abernethy AP. Coping with sexual concerns after cancer: the use of flexible coping. Support Care Cancer. 2010;18(7):785-800.

32. Ljungman L, Ahlgren J, Petersson LM, Flynn KE, Weinfurt K, Gorman JR, et al. Sexual dysfunction and reproductive concerns in young women with breast cancer: type, prevalence, and predictors of problems. Psychooncology. 2018;27(12):2770-7.

33. Ruddy KJ, Gelber SI, Tamimi RM, Ginsburg ES, Schapira L, Come SE, et al. Prospective study of fertility concerns and preservation strategies in young women with breast cancer. J Clin Oncol. 2014;32(11):11516.
34. Vuković P, Kasum M, Raguž J, Lonjak N, Knežević SB, Orešković l, et al. Fertility preservation in young women with early-stage breast cancer. Acta Clin Croat. 2019;58(1):147-56.

35. Partridge AH, Ruddy KJ, Barry WT, Greaney ML, Ligibel JA, Sprunck-Harrild $\mathrm{KM}$, et al. A randomized study to improve care for young women with breast cancer at community and academic medical oncology practices in the United States: the Young and Strong study. Cancer. 2019;125(11):1799-806.

36. Maclaran K, Panay N. Current concepts in premature ovarian insufficiency. Womens Health. 2015;11(2):169-82.

37. Aerts L, Christiaens MR, Enzlin P, Neven P, Amant F. Sexual functioning in women after mastectomy versus breast conserving therapy for early-stage breast cancer: a prospective controlled study. Breast. 2014; 23(5):629-36.

38. Miaja M, Platas A, Martinez-Cannon BA. Psychological impact of alterations in sexuality, fertility, \& body image in young breast cancer patients and their partners. Rev Investig Clin. 2017;69(4):204-9.

39. Sociedade Portuguesa de Ginecologia. Cancro ginecológico: consensos nacionais 2020 [homepage]. Lisboa: SPG; 2020. Available from: https:// spginecologia.pt/consenso/8252/

40. Stabile C, Gunn A, Sonoda Y, Carter J. Emotional and sexual concerns in women undergoing pelvic surgery and associated treatment for gynecologic cancer. Transl Androl Urol. 2015;4(2):169-85.

41. Fahami F, Mohamadirizi S, Savabi M. The relationship between sexual dysfunction and quality of marital relationship in genital and breast cancers women. J Educ Health Promot. 2017;6:56.

42. Kim SI, Lee Y, Lim MC, Joo J, Park K, Lee DO, et al. Quality of life and sexuality comparison between sexually active ovarian cancer survivors and healthy women. J Gynecol Oncol. 2015;26(2):148-54.

43. Onujiogu N, Johnson T, Seo S, Mijal K, Rash J, Seaborne L, et al. Survivors of endometrial cancer: who is at risk for sexual dysfunction? Gynecol Oncol. 2011;123(2):356-9.

44. Damast S, Alektiar KM, Goldfarb S, Eaton A, Patil S, Mosenkis J, et al. Sexual functioning among endometrial cancer patients treated with adjuvant high-dose-rate intra-vaginal radiation therapy. Int J Radiat Oncol Biol Phys. 2012;84(2):e187-93.

45. International Clinical Guideline Group. International guidelines on vaginal dilation after pelvic radiotherapy. Oxon: National Forum of Fynaecological Oncology Nurses; 2012.

46. Yang X, Rossi P, Bruner DW, Tridandapani S, Shelton J, Liu T. Noninvasive evaluation of vaginal fibrosis following radiotherapy for gynecologic malignancies: a feasibility study with ultrasound B-mode and Nakagami parameter imaging. Med Phys. 2013;40(2):022901.

47. QuickAM, Seamon LG,Abdel-Rasoul M, Salani R, Martin D. Sexual function after intracavitary vaginal brachytherapy for early-stage endometrial carcinoma. Int J Gynecol Cancer. 2012;22(4):703-8.

48. Jensen PT, Groenvold M, Klee MC, Thranov I, Petersen MA, Machin D. Longitudinal study of sexual function and vaginal changes after radiotherapy for cervical cancer. Int J Radiat Oncol Biol Phys. 2003;56(4):93749.

49. Bakker RM, Mens JW, de Groot HE, Tuijnman-Raasveld CC, Braat C, Hompus WC, et al. A nurse-led sexual rehabilitation intervention after radiotherapy for gynecological cancer. Support Care Cancer. 2017;25(3): 729-37. 
50. Jefferies H, Hoy S, McCahill R, Crichton A. Guidelines on vaginal dilator use after pelvic radiotherapy. Nurs Times. 2007;103(30):28-9.

51. Rosenberg SM, Tamimi RM, Gelber S, Ruddy KJ, Bober SL, Kereakoglow $\mathrm{S}$, et al. Treatment-related amenorrhea and sexual functioning in young breast cancer survivors. Cancer. 2014;120(15):2264-71.

52. Hanlon A, Small Jr W, Strauss J, Lin LL, Hanisch L, Huang L, et al. Dilator use following vaginal brachytherapy for endometrial cancer: a randomized feasibility and adherence study. Cancer Nurs. 2018;41(3):2009.

53. International Agency for Research on Cancer. Estimated aged-standardized incidence rates (world) in 2020, worlwide, females, all ages [homepage]. IARC; 2020. Available from: https://gco.iarc.fr/today/online-analysis-multi-bars? $v=2018 \&$ mode $=$ cancer $\&$ mode_population $=$ countries \&population $=900 \&$ populations $=900 \& k e y=a s r \& s e x=$ $2 \&$ cancer $=39 \&$ type $=0 \&$ statistic $=5 \&$ prevalence $=0 \&$ population_group $=0 \&$ ages_group $\% 5 \mathrm{~B} \% 5 \mathrm{D}=0$ \&ages_group $\% 5 \mathrm{~B} \% 5 \mathrm{D}=17 \&$ nb_items $=$ 10\&

54. Graziottin A. Effect of premature menopause on sexuality. Womens Health. 2007;3(4):455-74.

55. Kaltenecker B, Tikaria R. Vaginal cancer [Internet]. Treasure Island: StatPearls Publishing; 2021. Available from: https://www.ncbi.nlm.nih.gov/ books/NBK559126/

56. di Donato V, Bellati F, Fischetti M, Plotti F, Perniola G, Paniciet PB. Vaginal cancer. Crit Rev Oncol Hematol. 2012;81(3):286-95.

57. Ling B, Gao Z, Sun M, Sun F, Zhang A, Zhao W, et al. Laparoscopic radical hysterectomy with vaginectomy and reconstruction of vagina in patients with stage I of primary vaginal carcinoma. Gynecol Oncol. 2008;109(1):92-6.

58. Chamorro T. Cancer of the vulva and vagina. Semin Oncol Nurs. 1990;6(3):198-205.

59. Merino MJ.Vaginal cancer: the role of infectious and environmental factors. Am J Obstet Gynecol. 1991;165(4 Pt 2):1255-62.

60. Faubion SS, Kuhle CL, Shuster LT, Rocca WA. Long-term health consequences of premature or early menopause and considerations for management. Climateric. 2015;18(4):483-91.
61. Koothirezhi R, Ranganathan S. Postmenopausal syndrome [Internet]. Treasure Island: StatPearls Publishing; 2021. Available from: https:// www.ncbi.nlm.nih.gov/books/NBK560840/

62. Lind $H$, Waldenström AC, Dunberger $G$, al-Abany M, Alevronta $E$, Johansson $\mathrm{KA}$, et al. Late symptoms in long-term gynaecological cancer survivors after radiation therapy: a population-based cohort study. $\mathrm{Br}$ J Cancer. 2011;105(6):737-45.

63. Rebelo L. Intervenção breve no paciente fumador: aplicação prática dos '5 AS' e dos ' 5 RS' [Brief intervention with smokers: practical application of '5 AS' and the '5 RS']. Rev Port Med Geral Fam. 2011;27(4):35661. Portuguese

64. Clinical Practice Guideline Treating Tobacco Use and Dependence 2008 Update Panel, Liaisons, and Staff. A clinical practice guideline for treating tobacco use and dependence: 2008 update - A U.S. Public Health Service Report. Am J Prev Med. 2008;35(2):158-76.

65. Trinkaus M, Chin S, Wolfman W, Simmons C, Clemons M. Should urogenital atrophy in breast cancer survivors be treated with topical sstrogens? Oncologist. 2008;13(3):222-31.

66. Schover LR. Counseling cancer patients about changes in sexual function. Oncology. 1999;13(11):1585-91.

67. Annon JS. The PLISSIT model: a proposed conceptual scheme for the behavioral treatment of sexual problems. J Sex Educ Ther. 1976;2(1):115.

\section{CONFLITO DE INTERESSES}

A autora declara não possuir quaisquer conflitos de interesse.

\section{ENDEREÇO PARA CORRESPONDÊNCIA}

Maria Beatriz Morgado

E-mail: mbmorgado@campus.ul.pt

https://orcid.org/0000-0002-0000-1586

Recebido em 20-05-2020

Aceite para publicação em 15-02-2021 


\section{ABSTRACT}

\section{SEXUAL PROBLEMS IN WOMEN WITH BREAST CANCER AND GYNAECOLOGICAL CANCER: A NARRATIVE REVIEW}

Objectives: It is estimated that half of the women with breast cancer or gynecological cancer have sexual problems. However, only a minority receive medical advice in this area. This narrative review aims to describe the main sexual problems of women with this typology of neoplasia and to identify strategies to address and treat them.

Methods: A literature search of the PubMed database using the MeSH terms: 'Sexuality', 'Neoplasms', 'Female' and 'Women' has been performed. Articles and books dated from 1998 to 2020 were used.

Results: Breast cancer affects sexual health since diagnosis, due to psychological suffering. Additionally, it is important to consider the impact of the treatment. The surgery alters self-image, breast sensitivity, and mobility. Radiotherapy alters the properties of the tissues. Chemotherapy has psychological and physical effects with direct and indirect impacts on sexuality. Hormonal therapy leads to urogenital atrophy and vasomotor symptoms. On the other hand, most women with gynaecological cancer also experience sexual dysfunction secondary to therapy. Recent treatments such as brachytherapy, modified nerve-sparing techniques, and reconstructive surgery have contributed to a decrease in morbidity associated with cancer treatment. There is a growing awareness of the importance of sexual health in cancer patients. The doctor should inform patients about the possible impact of the disease and its treatment on their sexuality. There are validated questionnaires and models that facilitate communication. It is also the clinicians' responsibility to control the symptoms of sexual dysfunction associated with oncological therapy, favouring drugs with a neutral or positive impact on sexuality.

Conclusion: Any physician should be aware of sexual dysfunction associated with cancer and its treatment, being able to identify sexual dysfunctions, and adapt the therapeutic plan according to the cancer care continuum.

Keywords: Sexuality; Oncology; Neoplasia; Women. 